\title{
A NOVEL ALGORITHM FOR IMAGE DENOISING USING DT-CWT
}

\author{
SK.Umar Faruq ${ }^{1}$, Dr.K.V.Ramanaiah ${ }^{2}$, Dr.K.Soundararajan ${ }^{3}$ \\ ${ }^{1}$ Department of Electronics \& Comminications, QCET Nellore, A.P, India \\ ${ }^{2}$ Department of Electronics \& Comminications, Y.S.R College, Proddutur, A.P, India \\ ${ }^{3}$ Department of Electronics \& Comminications , TKR Engineering College, \\ Hyderabad, T.S, India
}

\begin{abstract}
This paper addresses image enhancement system consisting of image denoising technique based on Dual Tree Complex Wavelet Transform (DT-CWT). The proposed algorithm at the outset models the noisy remote sensing image (NRSI) statistically by aptly amalgamating the structural features and textures from it. This statistical model is decomposed using DTCWT with Tap-10 or length-10 filter banks based on Farras wavelet implementation and sub band coefficients are suitably modeled to denoise with a method which is efficiently organized by combining the clustering techniques with soft thresholding - softclustering technique. The clustering techniques classify the noisy and image pixels based on the neighborhood connected component analysis(CCA), connected pixel analysis and inter-pixel intensity variance (IPIV) and calculate an appropriate threshold value for noise removal. This threshold value is used with soft thresholding technique to denoise the image. Experimental results shows that that the proposed technique outperforms the conventional and state-of-the-art techniques.It is also evaluated that the denoised images using DTCWT (Dual Tree Complex Wavelet Transform) is better balance between smoothness and accuracy than the DWT.. We used the PSNR (Peak Signal to Noise Ratio) along with RMSE to assess the quality of denoised images.
\end{abstract}

\section{KEYWORDS}

Image Denoising, DTCWT, Tap-10 Filter banks, Soft-Clustering, PSNR

\section{INTRODUCTION}

The imaging devices which acquire or process satellite images introduce some negativeartifacts in the images. The images composed by different type of Devices are generally contaminated by different types of noise[1]. Image denoising is done in prior to image processing techniques. There are different types of noises that affect the digital images like Shot noise, amplifier noise and quantization noise and Speckle noise. In general noise characteristics in an image depends on many factors, which comprise sensor type, pixel dimensions, temperature, exposure time, and ISO speed [2].Image coefficients concentrate on low frequency components whereas the noise has both low as well as high frequency components. The high-frequency components can easily be removed, whereas it is a challenging task to eliminate low frequency noise as it is difficult to distinguish between real data and low-frequency noise. Most of the natural images are assumed to have additive white Gaussian noise. Speckle noise [3] is observes in satellite images. Thus, 
Signal \& Image Processing : An International Journal (SIPIJ) Vol.8, No.3, June 2017

denoising is the initial step to be considered before analyzing the image data. An efficient denoising technique is used to compensate for any data corruption. The objective of denoising is to remove the noise while preserving the important image details as much as possible. The most common technique used in the denoising process is the Bilateral filtering techniques [4][5]. It assist in to sustain the edge details by suppressing only the noisy coefficients. For a noisy image, there are some differences between the coefficients of original image and noise, the components that correspond to noise will be distributed among low magnitude high frequency components. Most of the low frequency noisy components are similar to image detail. In this way, the best way to take out those noises is carried out by comparing all the coefficients with a threshold and cutting off the coefficients that have smaller values than the limits [6]. Wavelet thresholding is the best strategy. In Wavelet thresholding, image is decomposed into approximation (lowfrequency) and detail (high-frequency ) sub bands, and the coefficients in the detail sub bands are processed via hard or soft thresholding [6][7]. The hard thresholding removes (sets to zero) coefficients that are smaller than a thresholding, the soft thresholding shrinks the coefficients that are larger than the threshold as well. The main objective of the wavelet thresholding is the selection of threshold value and the effect of denoising depends on the selected threshold; a bigger threshold will throw off the useful information and the noise components at the same time, while a smaller threshold cannot eliminate the noise effectively.Hence the proper selection of the threshold value is a most difficult task, and that can effectively obstructs the loss of significant image information. As a matter of fact noise components are characterized by high intensity (high frequency) distribution, where as image components are by low and medium intensity (low and medium frequency) values. The soft thresholding operation conditioned by the threshold parameter can effectively remove the high frequency noise components, but it cannot remove the low and medium frequency noise components which are merged with the image components. This is due to the fact that it is highly difficult to provide the precise value of threshold parameter value to remove these medium and low frequency noise components merged with the image features. Donoho [8]gave a general estimation method of threshold, In [9]Change et alhave used predictive models to estimate the threshold. It is a spatially adaptive threshold based on context modeling. In addition to this data-driven threshold for image denoising in a Bayesian frame work [10] has been proposed. but it may not be efficient up to the expected level.

In order to remove all noise components completely without losing the significant image information, a threshold value is designed using clustering techniques based on CCA,CPA and IPIV and suitably optimized to make it appropriate. This appropriate threshold value can remove all noise components efficiently when employed as parameter in soft thresholding.

In the recent years there has been a fair amount of research on wavelet based image denoising, because wavelet provides an appropriate basis for image denoising. But this single tree wavelet based image denoising has poor directionality, loss of phase information and shift sensitivity [11] as its limitations. Hence we proposed dual tree wavelet structure for an improved quality image denoising while getting rid of all above limitations.

This paper is organized as follows: section II describes the basic idea about the wavelet decomposition of the image section III explain the denoising algorithm of this approach. Experimental result is presented in section IV, lastly the conclusion of this paper is outlined in section V. 
Signal \& Image Processing : An International Journal (SIPIJ) Vol.8, No.3, June 2017

\section{DUAL TREE FRAME WORK}

The dual-tree complex wavelet transform (DTCWT) has recently received significant importance in the wavelet domain, owing primarily to its directional selective [12] and near-shift invariant [11] properties. It has been shown that with two separate maximally decimated and dyadic decompositions [13] where filters are offset by a half sample, the resulting CWT wavelet bases form an approximate Hilbert transform pair[14]. It achieves this with a redundancy factor of only $2^{d}$ for $d$-dimensional signals, which is considerably lower than the un-decimated DWT. The multidimensional (M-D) dual-tree CWT is non-separable but is based on a computationally efficient, separable filter bank [15].The dual tree complex wavelet transform allows the perfect reconstruction using short linear phase filters[16], also provide efficient order-N computation only twice the simple DWT for 1-D.

In formulation the DTCWT [17] is a complex pair of real and imaginary discrete wavelet transform trees. The schematic structure of Kingsbury's DTCWT implementation is shown in Fig.(1). Since the single tree wavelet structure is descent in required directional selectivity in two or multiple directions and also due to its shift sensitivity, lack of phase information, the dual tree structure is proposed by kingsbury. The analysis and synthesis filter banks used in the proposed DTCWT framework are Length-10 filters based on Farras wavelet implementation. A separate set of analysis and synthesis filter banks are used for first stage and higher stages. The filter coefficients used for real and imaginary trees are shown in table(1).

An effective decomposition level must be determined since it is one of the most significant factor in the wavelet denoising. If the higher decomposition level is used, the thresholding can remove some significant coefficients of the original image; therefore, increasing the decomposition level excessively high will decrease the PSNR after an optimal level and also enhance the complexity of the decomposition. For this purpose, a noise added image is used to obtain how the performance is changing with respect to the decomposition level.

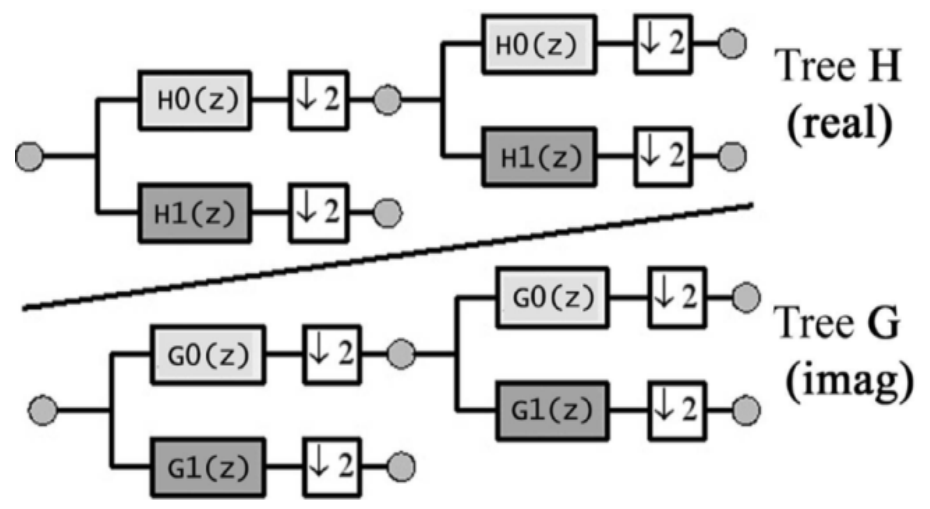

Fig 1:Kingsbury's dual-tree Complex WaveletTransform 
Signal \& Image Processing : An International Journal (SIPIJ) Vol.8, No.3, June 2017

Table1. Tap-10 Filter coefficients for DTCWT Framework.

\begin{tabular}{|r|r|r|r|}
\hline H0 & H1 & G0 & G1 \\
\hline 0 & 0 & 0.011226792 & 0 \\
\hline-0.088388348 & -0.011226792 & 0.011226792 & 0 \\
\hline 0.088388348 & 0.011226792 & -0.088388348 & -0.088388348 \\
\hline 0.695879989 & 0.088388348 & 0.088388348 & -0.088388348 \\
\hline 0.695879989 & 0.088388348 & 0.695879989 & 0.695879989 \\
\hline 0.088388348 & -0.695879989 & 0.695879989 & -0.695879989 \\
\hline-0.088388348 & 0.695879989 & 0.088388348 & 0.088388348 \\
\hline 0.011226792 & -0.088388348 & -0.088388348 & 0.088388348 \\
\hline 0.011226792 & -0.088388348 & 0 & 0.011226792 \\
\hline 0 & 0 & 0 & -0.011226792 \\
\hline
\end{tabular}

\section{RELATED WORK}

In this paper we take remote sensing image into special consideration for image denoising .In this attempt we modeled noisy remote sensing image statistically with textures and structures as shown in Fig (2) which is decomposed using a DTWCT to obtain the spectral domain coefficients .Texture analysis and texture extraction is aimed at improving the actual image content of interest from its background. Which can be helpful when objects in an image are more characterized by their texture than by intensity. Whereas the structural features are generally meant for highlighting the edge and ridge features so as to preserve the actual image boundaries from being washed out during the process of denoising. The main stages of the denoising method are shown in Fig 3.

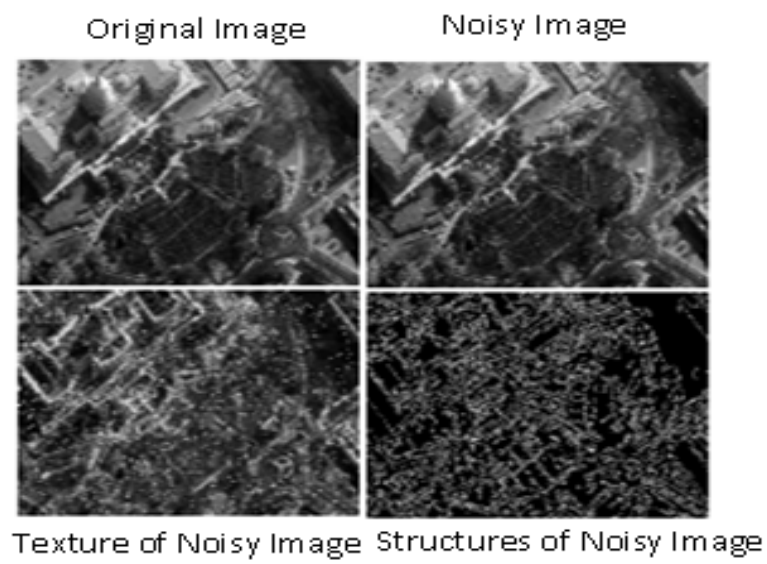

Fig 2. Textures and Structures of the noisy remote sensing image

To denoise the image under test we proposed a novel algorithm which is a feature diffused hybridization of the clustering based threshold techniques and soft thresholding. The performance of the Soft threshold technique is steered by the proper threshold value provided as input.Hence the proper selection of the threshold value is a most significant task, that can be effectively obstructs the loss of significant image information. The clustering based techniques, Otsu and Kapur put a closer insight into the Neighborhood Pixel Connectivity (NPC), Inter-Pixel 
Signal \& Image Processing : An International Journal (SIPIJ) Vol.8, No.3, June 2017

Connectivity (IPC),Inter-Pixel-Intensity-Variance (IPIV) and Connected Component Analysis (CCA) in order to cluster the noisy and image pixels in a view to design an appropriate threshold for denoising. The proposed Soft-Clustering method is a Spectro-spatial process, in which thresholdis estimated in spatial domain and denoising is performed in spectral domain. Thus SoftClustering process includes three sub-tasks viz (i) Threshold design, (ii) Threshold optimization and (iii) Soft thresholding.

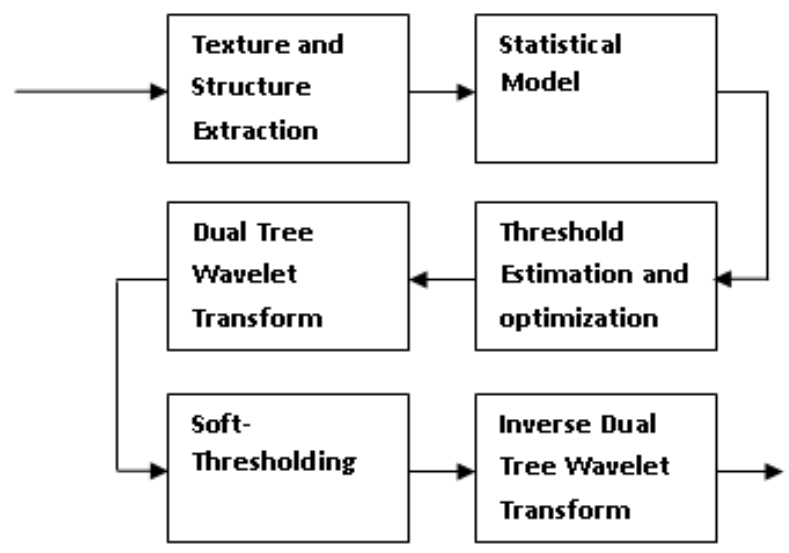

Fig 3. Diagram of the proposed denoising method

\subsection{Threshold Design}

Each object in the image is characterized by an intensity region, which consists of a group of pixels with similar intensity values and hence all these pixels are said to be connected to each other. The pixel inter-relationships and connectivity are represented by the terms neighborhood, adjacency, connectivity, connected paths, regions and boundaries. Considering these inter- pixel relationships as basis and using IPIV and CCA, the clustering technique designs an appropriate threshold value as follows. If $\mathrm{f}(\mathrm{i}, \mathrm{j})$ is the target pixel ,contaminated by noise $\mathrm{n}(\mathrm{i}, \mathrm{j})$, resulting the corrupted target pixel $\hat{f}(i, j)$ is given as $\hat{f}(i, j)=f(i, j)+n(i, j)$. Let us consider a $5 \mathrm{X} 5$ neighborhood of $\hat{f}(i, j)$ as in Fig(4). and compute deviation in pixel intensity of $\hat{f}(i, j)$ from the adjacent pixels as pixel intensity difference ' $d_{i}$ ' represented as

$$
\begin{aligned}
& d_{1}=\hat{f}(i, j)-f(i, j-1) \quad d_{2}=\hat{f}(i, j)-f(i, j+1), d_{3}=\hat{f}(i, j)-f(i-1, j), \\
& d_{4}=\hat{f}(i, j)-f(i+1, j), \quad d_{5}=\hat{f}(i, j)-f(i-1, j-1), d_{6}=\hat{f}(i, j)-f(i-1, j+1), d_{7}= \\
& \hat{f}(i, j)-f(i+1, j-1), \quad d_{8}=\hat{f}(i, j)-f(i+1, j+1) \ldots \ldots \ldots \\
& \cdot d_{24}=\hat{f}(i, j)-f(i+1, j+2), d_{25}=\hat{f}(i, j)-f(i+2, j+2)
\end{aligned}
$$

further these pixel differences are interpolated [18]with proper weights $\alpha_{i, j}$, as shown in Eq.(3) which is obtained from the basis of neighborhood pixel distances ,in order to design an appropriate threshold for image denoising. For a 5X5 neighborhood, assumed earlier, the distance weight matrix $\alpha_{i, j}$, is 
Signal \& Image Processing : An International Journal (SIPIJ) Vol.8, No.3, June 2017

$$
\alpha_{i, j}=\alpha[i][j]=\left[\begin{array}{lllll}
2 & 2 & 2 & 2 & 2 \\
2 & 1 & 1 & 1 & 2 \\
2 & 1 & 0 & 1 & 2 \\
2 & 1 & 1 & 1 & 2 \\
2 & 2 & 2 & 2 & 2
\end{array}\right]
$$

The interpolated threshold value

$$
\text { Thre }_{\text {inter }}=\sum_{i=1}^{m} \sum_{j=1}^{n} \alpha_{i j} d_{i j} .
$$

The gray level standard deviation ' $\sigma$ ', and for ' $\mathrm{N}$ ' iterations, the normalized threshold over the neighborhood is given by

$$
\text { Thre }_{\text {norm }}=\text { Thre }_{\text {inter }} /(\text { rowindex } * \text { colindex }) .
$$

Where row-index and col-index represent the size of the neighborhood considered. The threshold norm is further modified according to the range of the IPIV, ' $\sigma$ ' and ' $N$ ' to get the truncated threshold norm. Basis for this threshold setting is segmenting the image pixles into n-regions, characterized by the conditions given as (a) $\bigcup_{i=1}^{n} R_{i}=D,(\mathrm{~b}) R_{i} \cap R_{j}=\emptyset$, (c)ident $\left(R_{i}\right)=$ true, (iv)ident $\left(R_{j}\right)=$ false (v)ident $\left(\left(R_{i} \cup R_{j}\right)=\right.$ false, where ' $\mathrm{D}$ ' being the spatial domain in which the image is defined and 'ident' is the property of gray level similarity between the pixels in the region.All image regions will be having almost similar gary level distribution, but the noisy pixel regions have an extremely different gray level distribution and hence they seems to be an odd man that has to be restored .

\begin{tabular}{|c|c|c|c|c|}
\hline$f(i-2, j-2)$ & $f(i-2, j-1)$ & $f(i-2, j)$ & $f(i-2, j+1)$ & $f(i-2, j+2)$ \\
\hline$f(i-1, j-2)$ & $f(i-1, j-1)$ & $f(i-1, j)$ & $f(i-1, j+1)$ & $f(i-1, j+2)$ \\
\hline$f(i, j-2)$ & $f(i, j-1)$ & $\widehat{\boldsymbol{f}}(\boldsymbol{i}, \boldsymbol{j})$ & $f(i, j+1)$ & $f(i, j+2)$ \\
\hline$f(i+1, j-2)$ & $f(i+1, j-1)$ & $f(i+1, j)$ & $f(i+1, j+1)$ & $f(i+1, j+2)$ \\
\hline$f(i+2, j-2)$ & $f(i+2, j-1)$ & $f(i+2, j)$ & $f(i+2, j+1)$ & $f(i+2, j+2)$ \\
\hline
\end{tabular}

Fig 4. A 5 X5 neighborhood of the corrupted pixel.

\subsection{Threshold Optimization.}

To optimize the threshold designed consider the pure image pixels region to be $R_{1}$ and the corrupted noisy pixel region to be $R_{2}$, with gray level mean $\mu_{1}$ and $\mu_{2}$ respectively.The gray level deviation in region $R_{2}$ may be due to different kind of noise from a set of Impulse, AWGN, poisson and speckle noise[19] models with zero mean and standard deviation ' $\sigma^{\prime}$. Assume the probability that a pixel of the image belongs to $R_{1}$ is $P_{1}$ and that of $R_{2}$ is $P_{2}$. The threshold value for clustering the noisy and image pixels is optimized as follows.Suppose the gray level in the image is denoted by ' $\mathrm{z}$ ', then

pdf of gray level in $R_{1}$ is

$$
p_{1}(z)=\frac{1}{\sqrt{2 \pi \sigma}} \exp \left(\frac{-\left(z-\mu_{1}\right)^{2}}{2 \sigma^{2}}\right)
$$

and $\mathrm{R} 2$ is 
Signal \& Image Processing : An International Journal (SIPIJ) Vol.8, No.3, June 2017

$$
p_{2}(z)=\frac{1}{\sqrt{2 \pi \sigma}} \exp \left(\frac{-\left(z-\mu_{2}\right)^{2}}{2 \sigma^{2}}\right) \text { (gausian distribution) }
$$

pdf of gray level for the entire image is

$p(z)=P_{1} p_{1}(z)+P_{2} p_{2}(z)(6)$

let us assume that $\mu_{1}<\mu_{2}$.Then the threshold ' $\mathrm{T}$ ' that maps the pixel to $R_{1}$ (if $z \leq T$ ) or to $R_{2}$ (if $z>T$ ), must satisfy $\mu_{1}<T<\mu_{2}$ as it distinguishes between the pixels of $R_{1}$ and $R_{2}$. Now the probability of erroneously classifying a noisy pixel (that actually belongs to $R_{2}$ ) to $R_{1}$ is

$$
E_{1}(T)=\int_{-\infty}^{T} p_{2}(z) d z
$$

Similarly the probability of erroneously classifying a pixel (that actually belongs to $R_{1}$ ) to $R_{2}$ is

$$
E_{2}(T)=\int_{T}^{\infty} p_{1}(z) d z=1-\int_{-\infty}^{T} p_{1}(z) d z
$$

The total error due to said threshold is

$$
E(T)=P_{2} E_{1}(T)+P_{1} E_{2}(T) .
$$

To find the optimum threshold value ,i.e. the threshold for which $E(T)$ is minimum,we differentiate $E(T)$ with respect to ' $\mathrm{T}$ ' and equate the result to zero,i.e.

$$
\begin{aligned}
\frac{d E}{d T}=\frac{d}{d T} P_{2} \int_{-\infty}^{T} p_{2}(z) d z+\frac{d}{d T} P_{1}\left[1-\int_{-\infty}^{T} p_{1}(z) d z\right]=0 \\
P_{2} p_{2}(T)-P_{1} p_{1}(T)=0 \\
P_{2} \frac{1}{\sqrt{2 \pi \sigma}} \exp \left(\frac{-\left(T-\mu_{2}\right)^{2}}{2 \sigma^{2}}\right)=P_{1} \frac{1}{\sqrt{2 \pi \sigma}} \exp \left(\frac{-\left(T-\mu_{1}\right)^{2}}{2 \sigma^{2}}\right) \\
\frac{P_{2}}{P_{1}} \frac{1}{\sqrt{2 \pi \sigma}} \exp \left(\frac{-\left(T-\mu_{2}\right)^{2}}{2 \sigma^{2}}\right)=\frac{1}{\sqrt{2 \pi \sigma}} \exp \left(\frac{-\left(T-\mu_{1}\right)^{2}}{2 \sigma^{2}}\right)
\end{aligned}
$$

Taking logarithm

$$
\begin{aligned}
& \ln \frac{P_{2}}{P_{1}}-\frac{\left(T-\mu_{2}\right)^{2}}{2 \sigma^{2}}=-\frac{\left(T-\mu_{1}\right)^{2}}{2 \sigma^{2}} \\
& 2 \sigma^{2} \ln \frac{P_{2}}{P_{1}}=\left(T-\mu_{2}\right)^{2}-\left(T-\mu_{1}\right)^{2} \\
& 2 \sigma^{2} \ln \frac{P_{2}}{P_{1}}=2 T\left(\mu_{1}-\mu_{2}\right)-\left(\mu_{1}+\mu_{2}\right)\left(\mu_{1}-\mu_{2}\right) \\
& \quad \frac{2 \sigma^{2}}{\left(\mu_{1}-\mu_{2}\right)} \ln \frac{P_{2}}{P_{1}}=2 T-\left(\mu_{1}+\mu_{2}\right)
\end{aligned}
$$

Hence the optimum threshold value is 
Signal \& Image Processing : An International Journal (SIPIJ) Vol.8, No.3, June 2017

$$
T=\frac{\left(\mu_{1}+\mu_{2}\right)}{2}+\frac{\sigma^{2}}{\left(\mu_{1}-\mu_{2}\right)} \ln \frac{P_{2}}{P_{1}}
$$

Assuming different values of standard deviations of gray levels in $R_{1}$ and $R_{2}$ result in a more general expression for the optimum threshold value.Suppose $\sigma_{1}$ and $\sigma_{2}$ are the standard deviations of the gray levels associated with $R_{1}$ and $R_{2}$ respectively then

$$
T=\frac{b \pm \sqrt{b^{2}-4 a c}}{2 a}
$$

Where $a=\sigma_{1}{ }^{2}-\sigma_{2}{ }^{2}$

$$
\begin{gathered}
b=2\left(\mu_{2}{\sigma_{1}}^{2}-\mu_{1}{\sigma_{2}}^{2}\right) \\
c=\sigma_{1}{ }^{2} \mu_{2}{ }^{2}-{\sigma_{2}}^{2} \mu_{1}{ }^{2}-2{\sigma_{1}}^{2} \sigma_{2}{ }^{2} \ln \frac{\sigma_{1} P_{2}}{\sigma_{2} P_{1}}
\end{gathered}
$$

A plus or minus sign is selected with the view that ' $\mathrm{T}$ ' satisfies the constraint: $\mu_{1}<T<\mu_{2}$

\subsection{Soft Thresholding.}

Using the designed optimum threshold value ,the soft thresholding operation is performed on the modeled Noisy remote sensing image spectral coefficients to obtain the noise free image without losing the significant image information. The soft thresholding operation involves attenuation of the noisy intensities detected using the set threshold to image level. The thresholding of spectral coefficients is performed by

$$
\widehat{W}(y)=\left(\begin{array}{c}
y+T ; y<-T \\
0 ;-T \leq y \leq T, \\
y-T ; T<y
\end{array},\right.
$$

where $\mathrm{T}$ is the designed threshold value.

The shape of the threshold curve is very much similar to the characteristics of a signum function. Hence the soft thresholding process is described by $\mathrm{Y}=\operatorname{sign}(\mathrm{X}) .(\mathrm{XI}-\mathrm{T})_{+},((\mathrm{X})+=0$ if $\mathrm{X}<0$; $(\mathrm{X})+$ $=\mathrm{X}$, if $\mathrm{X} \geq 0)$, and

$$
|| X|-T|=\left\{\begin{array}{c}
0 ; \text { if }(|X|-T) \\
(|X|-T) ; \text { for }(|X|-T)
\end{array}\right.
$$

where ' $\mathrm{X}$ ' is input noisy pixel intensity and ' $\mathrm{Y}$ ' is denoised output pixel intensity.

The denoised image is comparatively tested mathematically uaing quality metrics such as Peak Signal to Noise Ratio (PSNR) in decibels (dB), Root Mean Square Error(RMSE) to judge the quality of the proposed algorithm and the results are provided in results and discussion section. The Root Mean Square Error (RMSE) of the denoised image with respect to the noise free version is $R M S E(\hat{f})=\left(\frac{1}{N}\|f-\hat{f}\|^{2}\right)^{\frac{1}{2}}$. In denoising applications the image quality is often expressed in terms of PSNR which is defined as 
Signal \& Image Processing : An International Journal (SIPIJ) Vol.8, No.3, June 2017

$$
P S N R=10 \log _{10}\left(\frac{\operatorname{Max}\left(f^{2}\right)}{(\operatorname{RMSE}(\hat{f}))^{2}}\right)
$$

\section{Proposed algorithm:}

Step 1: Start with NRSI $y_{i, j}$; where $\mathrm{i}=\mathrm{m}, \mathrm{j}=\mathrm{n}$.

Step 2: Calculate textures and structures of NRSI.

$\operatorname{Text}(P, R)=\sum_{p=0}^{p-1} s\left(q_{p}-q_{c}\right) .2^{P}$, where P-pixel, R-Radius and S-unit step variation.

$\mathrm{L}(\mathrm{x}, \mathrm{y})=\frac{d^{2} I}{d x^{2}}+\frac{d^{2} I}{d y^{2}}$, where I:pixel intensity level.

Step 3:Model NRSI statistically with textures and structures.

$y_{i, j}=y_{i, j}+\operatorname{Text}(P, R)+L(x, y)$;

Where Text $(\mathrm{P}, \mathrm{R})$ Corresponds to textures and $\mathrm{L}(\mathrm{x}, \mathrm{y})$ corresponds to structures.

Step 4: Perform DT-CWT analysis of NRSI..

$$
W^{c}{ }_{i, j}=W^{r}{ }_{i, j}+W_{i, j}^{i}
$$

Step 5: Soft_clustering ()

\{

Threshold_design ()

\{

spot the Connected components and cluster the pixels using CCA;

$$
\begin{aligned}
& P_{i, j} / P_{i, j} \in P_{T} ; P_{T}: \text { Pixel threshold intensity. } \\
& P_{i, j} \in R_{k}: \text { if } P_{i, j}=P_{T} ; P_{i, j} \in R_{l}: \text { if } P_{i, j} \neq P_{T}
\end{aligned}
$$
threshold;

Compute the pixel intensity deviations using IPIV and interpolate them to get the

$$
d_{i, j}=P_{i+f, j+g}-P_{i, j} ; T_{i, j}=\sum_{i, j} \propto_{i, j} d_{i, j}
$$

Normalize this threshold value over the neighbourhood and truncate it to get the actual threshold;

\}

$$
T_{i, j}=T_{i, j} /(m * n) ; T_{i, j}=\operatorname{ceil}\left(T_{i, j}\right)
$$

Threshold_optimization ()

\{

Analyze the pdf of noisy and image pixel regions;

Check an arbitrary pixel certainty for corruption;

$$
p(z)=P_{1} p_{1}(z)+P_{2} p_{2}(z) \text {. }
$$

Perform probabilistic analysis to optimize the threshold;

Compute optimized threshold from the above analysis as final;

\}

Softthresholding ()

\{

Perform soft thresholding with the optimized threshold as input;

\}

\}

Step 6:Perfrom hierarchical synthesis using IDTCWT.

step 7:Test the results and calculate the quality metrics. 
Signal \& Image Processing : An International Journal (SIPIJ) Vol.8, No.3, June 2017

\section{RESULTS AND DISCUSSION}

The proposed algorithm is implemented in the Matlab environment and simulated to test the satellite image of size 256X256. The DTCWT implementation is based on Tap-10 or Length-10 filters of Farras wavelet implementation upto 2 decomposition levels. The denoised results of the proposed algorithm are illustrated in Fig(5). We have also shown the resultant denoising images for different noise variances in Fig.(6) and Fig.(7).We have compared the experimental results of the proposed method with that of VisuShrink, SURE Shrink and Bayes Shrink in terms of the PSNR, RMSE and the results are presented in Table (2).The tabulation was also prepared for variance vs PSNR \& RMSE for DWT and DTCWT using Soft Clustering function for various levels is shown in table (3) \& (4).It is evident from the results given in Table (2)that our proposed method outperforms remarkably over the VisuShrink, SureShrink, and BayesShrink .The performance of proposed algorithm in terms of PSNR values are illustrated graphically in fig(8), fig $(9) \mathrm{g}(10)$
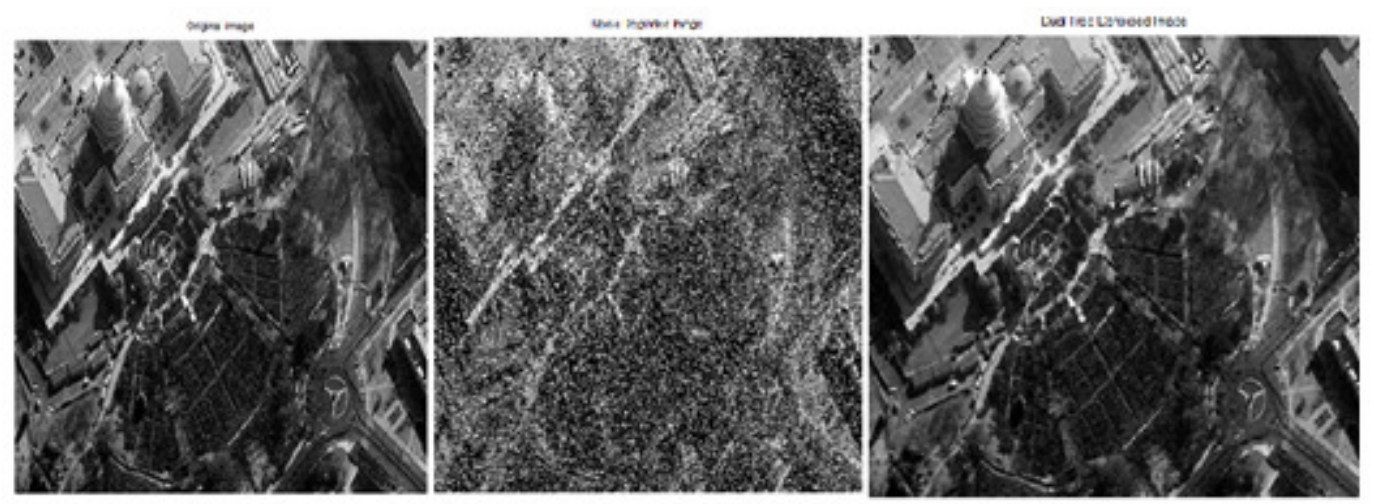

Fig 5: Denoising results of the proposed algorithm for white house image for noise of variance $=30$.
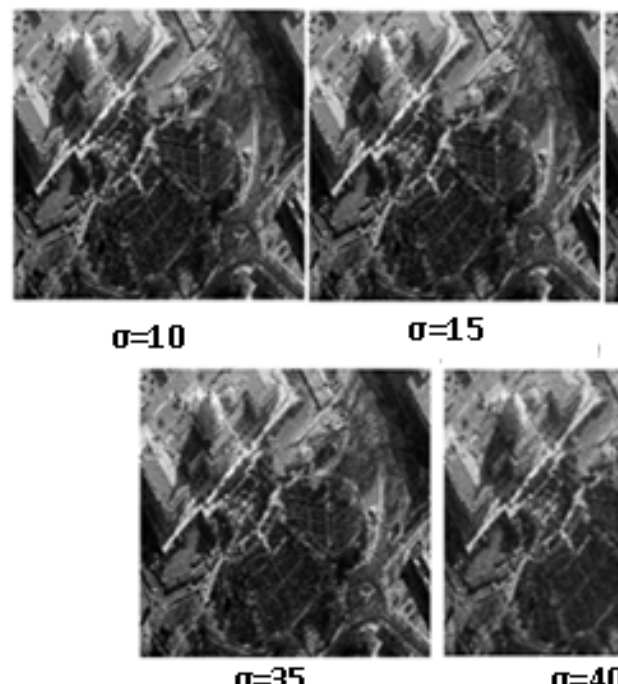

$\mathbf{0}=\mathbf{1 5}$

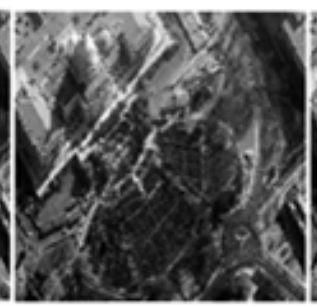

$\mathbf{0}=\mathbf{2 0}$

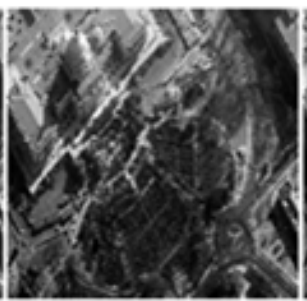

$\mathbf{\sigma}=\mathbf{2 5}$

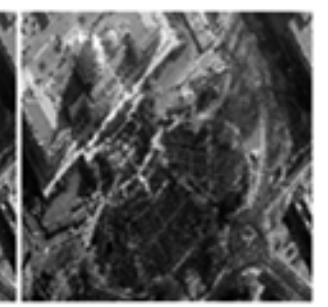

$\mathbf{0}=\mathbf{3 0}$

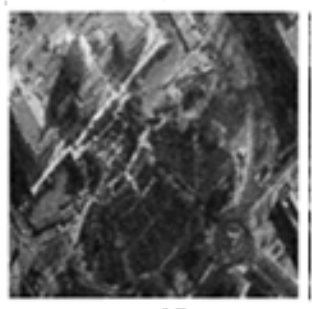

$\mathbf{\sigma}=\mathbf{4 0}$

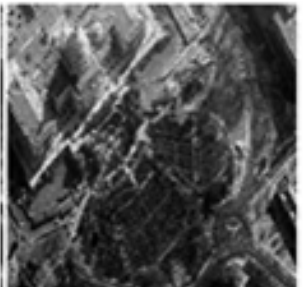

$\sigma=45$

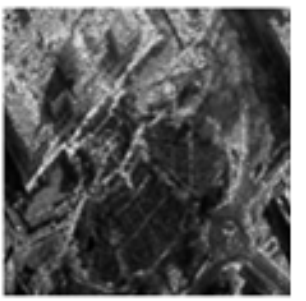

$\mathbf{0}=\mathbf{5 0}$

Fig 6: Denoising results of the proposed algorithm for white house image of different noise levels. 
Signal \& Image Processing : An International Journal (SIPIJ) Vol.8, No.3, June 2017
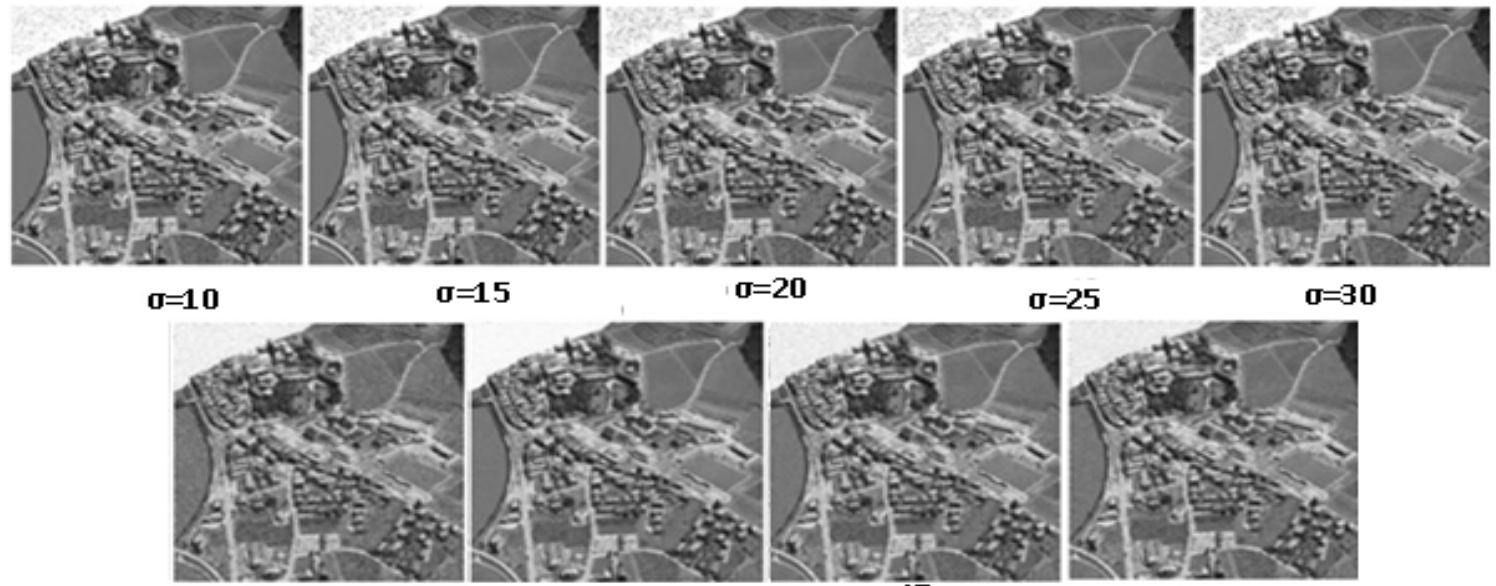

$\mathbf{\sigma}=\mathbf{1 5}$

$\mathbf{\sigma}=\mathbf{2 0}$

$\mathbf{\sigma}=25$

$\mathbf{\sigma}=\mathbf{3 0}$

$\mathbf{\sigma}=\mathbf{3 5}$

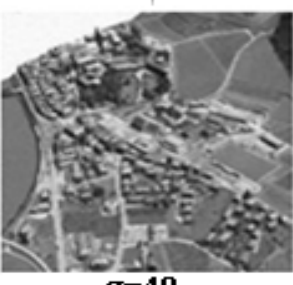

$\mathbf{\sigma}=\mathbf{4 0}$

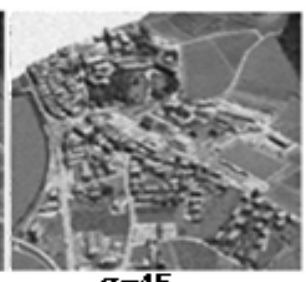

$\mathbf{\sigma}=\mathbf{4 5}$

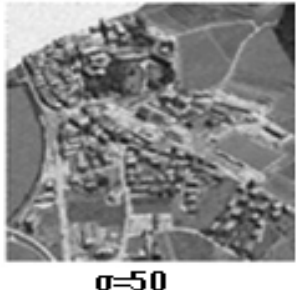

Fig 7: Denoising results of the proposed algorithm for geographic image of different noise levels.
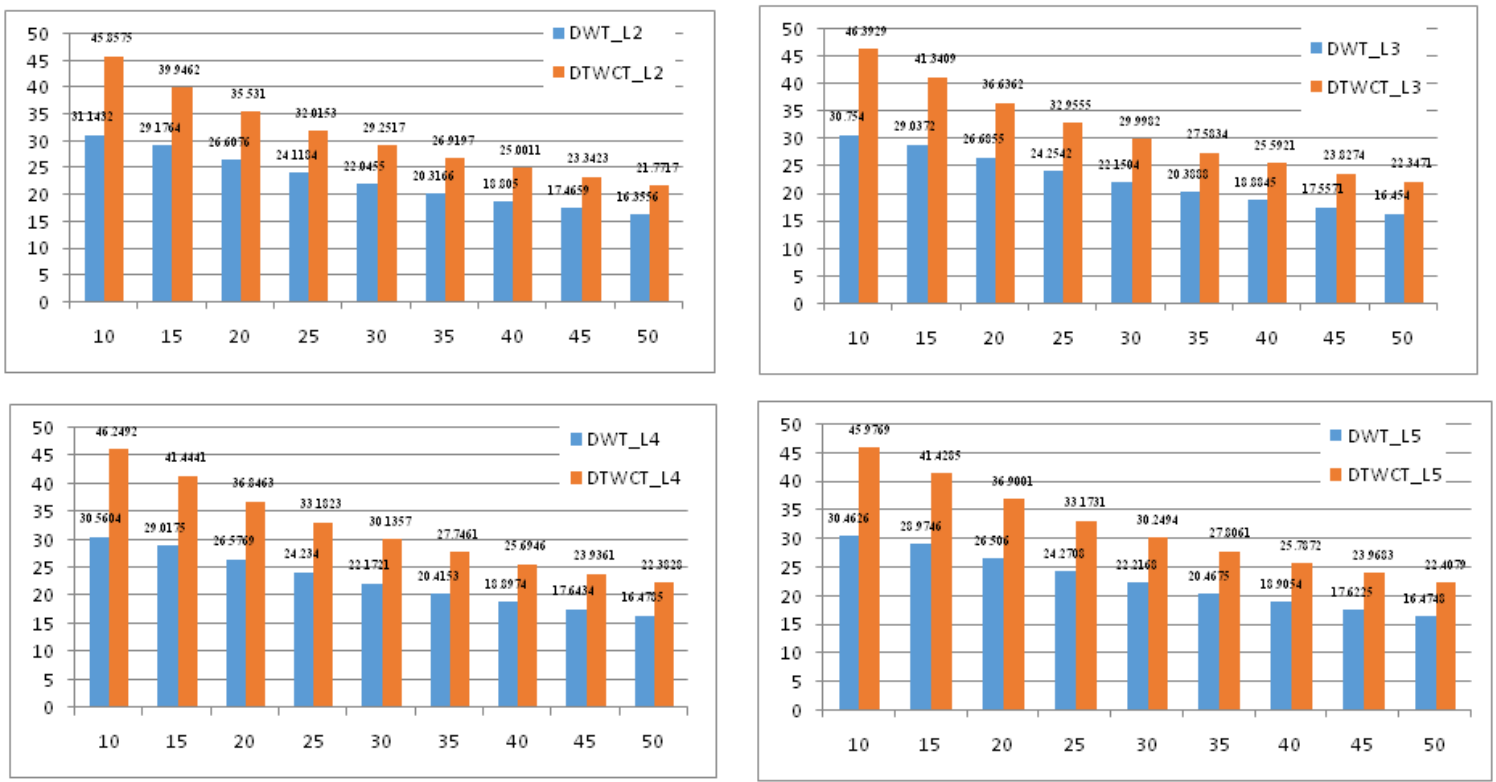

Fig(8):Bar-Graph comparison of PSNR values of the proposed algorithm with single and dual tree implementations of different levels.
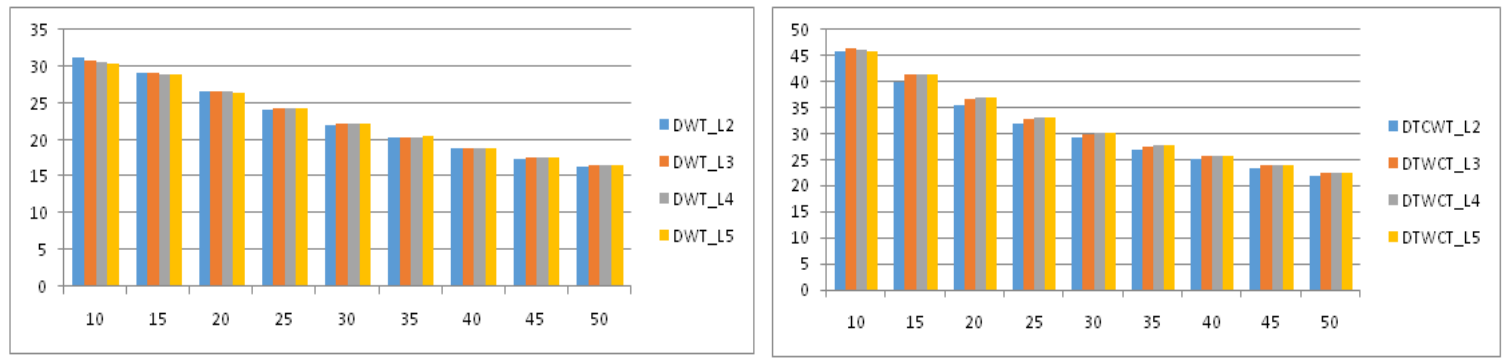

Fig(9):Bar-Graph comparison of PSNR values of DWT and DTCWT implementations of different levels 
Signal \& Image Processing : An International Journal (SIPIJ) Vol.8, No.3, June 2017

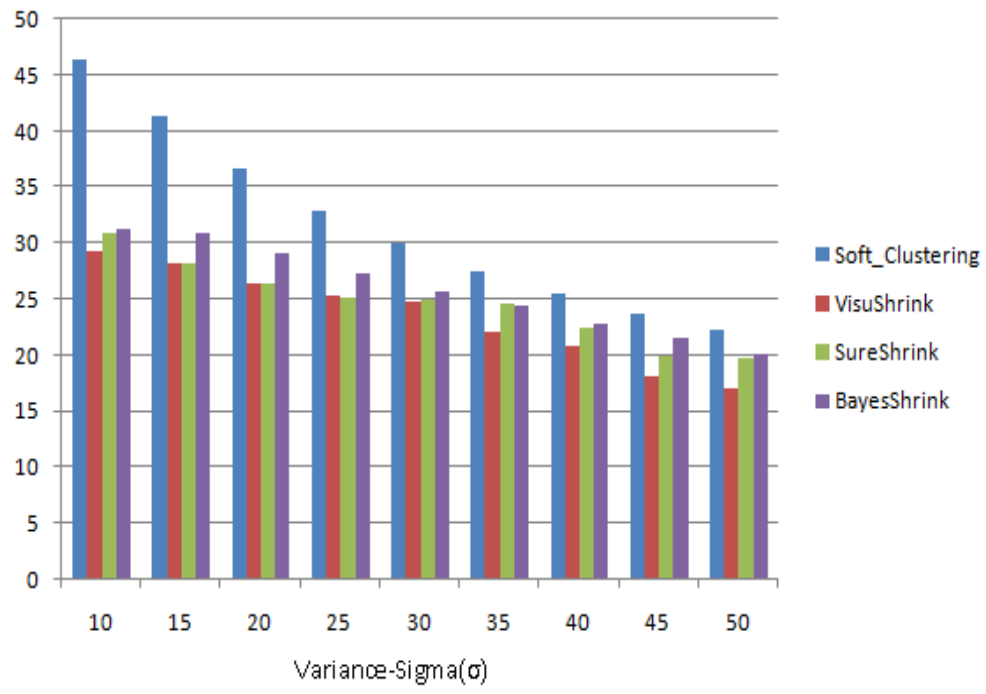

Fig (10):Bar-Graph comparison of PSNR values of proposed Soft-Clustering algorithm and other existing methods.

Table(2):PSNR comparison of the proposed algorithm with other existing methods.

\begin{tabular}{|c|c|c|c|c|}
\hline $\boldsymbol{\sigma}$ & Soft_Clustering & VisuShrink & Sureshrink & BayesShrink \\
\hline 10 & 46.3929 & 29.34 & 30.96 & 31.34 \\
\hline 15 & 41.3409 & 28.23 & 28.31 & 30.97 \\
\hline 20 & 36.6362 & 26.4 & 26.43 & 29.09 \\
\hline 25 & 32.9555 & 25.28 & 25.23 & 27.27 \\
\hline 30 & 29.9982 & 24.83 & 25.01 & 25.73 \\
\hline 35 & 27.5834 & 22.21 & 24.68 & 24.46 \\
\hline 40 & 25.5921 & 20.83 & 22.55 & 22.9 \\
\hline 45 & 23.8274 & 18.12 & 20.03 & 21.62 \\
\hline 50 & 22.3471 & 17 & 19.84 & 20.09 \\
\hline
\end{tabular}

Table(3):PSNR comparison of the proposed algorithm with Single tree and Dual tree implementations.

\begin{tabular}{|c|c|c|c|c|c|c|c|c|c|c|}
\hline \multirow{2}{*}{ Level } & \multirow{2}{*}{ Tool } & \multicolumn{9}{|c|}{ PSNR Value } \\
\hline & & $\sigma=10$ & $\sigma=15$ & $\sigma=20$ & $\sigma=25$ & $\sigma=\mathbf{3 0}$ & $\sigma=35$ & $\sigma=40$ & $\sigma=45$ & $\sigma=50$ \\
\hline \multirow{2}{*}{2} & DWT & 31.1432 & 29.1764 & 26.6076 & 24.1184 & 22.0455 & 20.3166 & 18.805 & 17.4659 & 16.3556 \\
\hline & DTCWT & 45.8575 & 39.9462 & 35.531 & 32.0153 & 29.2517 & 26.9197 & 25.0011 & 23.3423 & 21.7717 \\
\hline \multirow{2}{*}{3} & DWT & 30.754 & 29.0372 & 26.6855 & 24.2542 & 22.1504 & 20.3888 & 18.8845 & 17.5571 & 16.454 \\
\hline & DTCWT & 46.3929 & 41.3409 & 36.6362 & 32.9555 & 29.9982 & 27.5834 & 25.5921 & 23.8274 & 22.3471 \\
\hline \multirow{2}{*}{4} & DWT & 30.5604 & 29.0175 & 26.5769 & 24.234 & 22.1721 & 20.4153 & 18.8974 & 17.6434 & 16.4785 \\
\hline & DTCWT & 46.2492 & 41.4441 & 36.8463 & 33.1823 & 30.1357 & 27.7461 & 25.6946 & 23.9361 & 22.3828 \\
\hline \multirow{2}{*}{5} & DWT & 30.4626 & 28.9746 & 26.506 & 24.2708 & 22.2168 & 20.4675 & 18.9054 & 17.6225 & 16.4748 \\
\hline & DTCWT & 45.9769 & 41.4285 & 36.9001 & 33.1731 & 30.2494 & 27.8061 & 25.7872 & 23.9683 & 22.4079 \\
\hline
\end{tabular}


Signal \& Image Processing : An International Journal (SIPIJ) Vol.8, No.3, June 2017

Table (4):RMSE comparison of the proposed algorithm with Single tree and Dual tree implementations.

\begin{tabular}{|c|c|c|c|c|c|c|c|c|c|c|}
\hline \multirow{2}{*}{ LEVEL } & \multirow{2}{*}{ TOOL } & \multicolumn{9}{|c|}{ RMSE VALUE } \\
\hline & & $\sigma=10$ & $\sigma=15$ & $\sigma=20$ & $\sigma=25$ & $\sigma=30$ & $\sigma=35$ & $\sigma=40$ & $\sigma=45$ & $\sigma=50$ \\
\hline \multirow{2}{*}{2} & DWT & 7.057 & 8.8871 & 11.9238 & 15.9362 & 20.0562 & 24.702 & 29.1383 & 33.9227 & 38.7424 \\
\hline & DTCWT & 5.4371 & 9.3778 & 13.8771 & 18.5053 & 23.4458 & 28.5348 & 33.1129 & 38.1492 & 42.9474 \\
\hline \multirow{2}{*}{3} & DWT & 7.4105 & 8.9579 & 11.8538 & 15.7424 & 19.9076 & 24.3578 & 28.8994 & 33.4385 & 38.3328 \\
\hline & DTCWT & 5.2497 & 8.4464 & 12.613 & 17.2018 & 21.9511 & 26.6771 & 31.5991 & 36.4596 & 41.5072 \\
\hline & DWT & 7.5596 & 9.0581 & 11.949 & 15.6446 & 19.838 & 24.2394 & 29.0624 & 33.6278 & 38.3623 \\
\hline & DTCWT & 5.6075 & 8.579 & 12.661 & 17.1792 & 21.6926 & 26.5538 & 31.5111 & 36.4641 & 41.4059 \\
\hline & DWT & 7.6499 & 9.1103 & 12.0292 & 15.614 & 19.8733 & 24.2077 & 28.7361 & 33.5284 & 38.2745 \\
\hline & DTCWT & 5.7784 & 8.664 & 12.6212 & 17.0749 & 21.7665 & 26.5651 & 31.3395 & 36.4427 & 41.2791 \\
\hline
\end{tabular}

\section{CONCLUSION}

In this paper, we have developed an image denoising method based on adaptive Soft-Clustering technique for denoising the images efficiently. This method removes a large amount of additive noise and preserves most of the edges and visual quality of the image. As the denoising performance of soft threshold technique is conditioned by the aptness of the specified threshold parameter, a task to optimize the designed threshold value is done in the threshold optimization stage to make it appropriate for removing all high, medium and even low frequency noise components. Our proposed method gives better performance than the VisuShrink, SureShrink, and BayesShrink. A comparative analysis of denoising results is performed in tabular and graphical manner and provided to exploit the operational superiority of the proposed algorithm over the existing algorithms.

\section{REFERENCES}

[1] Mukesh C. Motwani, Mukesh C. Gadiya, Rakhi C. Motwani, Frederick C. Harris, Jr, 2004. Survey of Image DenoisingTechniques. Proc. of GSPx 2004, Santa Clara Convention Center, Santa Clara, CA, pp. 27-30.

[2] Ming Zhang and Bahadir K. Gunturk, 2008.Multiresolution Bilateral Filtering for Image Denoising.IEEE Transactions on Image Processing, Vol. 17, No. 12.

[3] Guo.H, J. E. Odegard, M. Lang, R. A. Gopinath, I.W. Selesnick and C. S. Burrus, 1994.Wavelet based Speckle Reduction with Application to SAR based ATD/R. First International Conference on Image Processing, 1, pp. 75-79.

[4] Canny.J, 1986 .A computational approach to edge detection. IEEE Trans.Pattern Anal. Machine Intell., vol. PAMI-8, pp. 679-697, Nov.

[5] Ming Zhang and Bahadir Gunturk, 2008.A New Image Denoising Method based on the Bilateral Filter.ICASSP, IEEE,pp. 929-932.

[6] Donoho.D.L 1995.De-noising by Soft Thresholding.IEEE Trans. on Inform, Theory, 41, No. 3, pp. 613- 627.

[7] Donoho.D..L and I. M. Johnstone, 1995.Adapting to Unknown Smoothness via Wavelet Shrinkage.Journal of the American Statistical Association, 90, No. 432, pp. 1200- 1224. 
Signal \& Image Processing : An International Journal (SIPIJ) Vol.8, No.3, June 2017

[8] Donoho.D..L and, I.M. Johnstone, 1994 "Ideal Spatial Adaptation by Wavelet Shrinkage," Biometrika, 81, No. 3, pp. 425-455

[9] Chang.S.G. et al. 2000.Adaptive Wavelet Thresholding for Image Denoising and Compression. IEEE Transactions on Image Processing, 9, pp. $1532-1546$.

[10] Chang,.S..G B. Yu, and M. Vetterli, 1998.Spatially Adaptive Wavelet Thresholding with Context Modeling for Image Denoising. Proc. ICIP, pp. 535-539.

[11] Kingsbury..N.G ,2001.Complex wavelets for shift invariant analysis and filtering of signals. Appl. Comput. Harmon. Anal., vol. 10, no. 3, pp.234(20)-253(20), May 2001.

[12] Van Spaendonck.R , F. Fernandes, M. Coates, and C. Burrus. 2000.Non-redundant, directionally selective, complex wavelets. In Proc. Int. Conf. Image Process. volume 2, pages 379\{382, Istanbul, Turkey, Sep. 2000.

[13] Vandergheynst.P and J.-F. Gobbers. 2002.Directional dyadic wavelet transforms: design and algorithms. IEEE Trans. Image Process., 11(4):363\{372, Apr. 2002.

[14] Selesnick. I.W 2001.Hilbert transform pairs of wavelet bases. Signal Process. Lett., 8(6):170\{173,Jun. 2001

[15] Herley.C and M. Vetterli. Wavelets and recursive filter banks.IEEE Trans. Signal Process., 41(8):2536.2556, 1993.

[16] Kingsbury. N..G ."The dual-tree complex wavelet transform: A new technique for shift invariance and directional filters," in Proc. IEEE DSP Workshop, Aug. 1998, Bryce, Canyon, paper no. 86.

[17] Fernandes, F.C.A ,R. L. C. van Spaendonck, and C. S. Burrus. A new framework for complex wavelet transforms. IEEE Trans. Signal Process., 51(7):1825\{1837, Jul. 2003.

[18] Tinku Acharya and Ping-Sing Tsai,2007 .Computational Foundations of Image Interpolation Algorithms, ACM Ubiquity, Vol. 8, 2007.

[19] Gagnon, L. 1999. Wavelet Filtering of Speckle Noise- some Numerical Results, Proceedings of the Conference Vision Interface, Trois-Reveres.

\section{AUTHOR}

Shaik. Umar Faruq is currently working as an Associate Professor \& Head in QUBA College of engineering and Technology, Nellore. He received B.E degree from Osmania University and M.Tech from JNTU in 2005 .since 2010 he has been a Ph.D student in the department of Electronics and Communications, JNTUA, Anantapur. He has 15 years of teaching experience both at UG and PG level and his research interests include Reconfigurable Architectures, Image and Video Processing

K.V. Ramanaiah is currently working as an Associate Professor \& Head in Yogi Vemana University, Kadapa. He received M.Tech degree from Jawaharlal Nehru Technological University, Hyderabad in 1998 and Ph.D degree from JNTUH in 2009. He has vast experience as academician and published number of papers in international Journals and conferences .His research interests include VLSI Architectures, Signal \& Image Processing. 
Signal \& Image Processing : An International Journal (SIPIJ) Vol.8, No.3, June 2017

K Soundara Rajan received the B.Tech in Electronics \& Communication Engineering from Sri Venkateswara University. M.Tech (Instrumentation \& Control) from Jawaharlal Nehru Technological University in 1972. Ph.D degree from University of Roorkee, U.P. He has published number of papers in international journals and conferences. He is a member of professional bodies like NAFEN, ISTE, IAENG etc,. He has vast experience as academician, administrator and philanthropist. He is reviewer for number of journals. His research interests include Fault Tolerant Design, Embedded Systems and signal processing. 